\section{THREE-DIMENSIONAL FIBRE PREFORM PROFILING}

Indexing terms: Optical fibres, Data processing

Results are reported of three-dimensional profiling of fibre preforms from deflection function measurements. We demonstrate the importance of performing the integration involved with a sufficient number of points and show how the systematic use of interpolation allows this number of points to be obtained from a few preform projections.

Introduction: Three-dimensional profiles of optical fibre preforms may be obtained from deflection function measurements by a procedure described recently by $\mathrm{Chu} .{ }^{1}$ This letter considers some aspects of the practical implementation of the reconstruction with emphasis on the data processing. We show that the systematic use of interpolation between the defiection angles corresponding to different preform orientations allows accurate three-dimensional profiles to be obtained from a relatively modest number of azimuthal projections. The usefulness of interpolation is illustrated with both simulated and measured profiles.

Measurement: The deflection data were obtained by the spatial filtering technique, ${ }^{2}$ in which a parallel beam of light illuminates the preform, immersed in an index-matching fluid; a rotating chopper allows the deflection angles to be measured with high accuracy in the time domain. The influence of the optical and electronic characteristics of the set-up on the resolution are reported elsewhere. ${ }^{3}$ The two external parameters of the procedure are the scan increment $\varepsilon$ and the number of projections $N_{\theta}$. In practice, $\varepsilon$ is chosen in the range 4-10 $\mu \mathrm{m}$, while $N_{\theta}$ depends on the azimuthal index variations in the preform.

Data processing: From Chu's results ${ }^{1}$ and under the assumption of small numerical aperture, the basic reconstruction equation for the normalised index difference can be expressed as follows:

$$
\frac{n(r, \psi)-n_{0}}{n_{0}}=\left.\frac{1}{2 \pi} \int_{-\pi / 2}^{\pi / 2} d \theta g(z, \theta)\right|_{z=r \sin (\psi-\theta)}
$$

where $g(z, \theta)$ is the convolution

$$
g(z, \theta)=\frac{1}{\pi} \int_{-\infty}^{\infty} \phi(\rho, \theta) \frac{d \rho}{z-\rho}
$$

$\phi(\rho, \theta)$ is the deflection angle for the scan position $\rho$ and the scan direction $\theta$, and $(r, \psi)$ are the polar co-ordinates of the preform cross-section.

If the preform is perfectly circular, the deflection measurement does not depend on the preform orientation and the

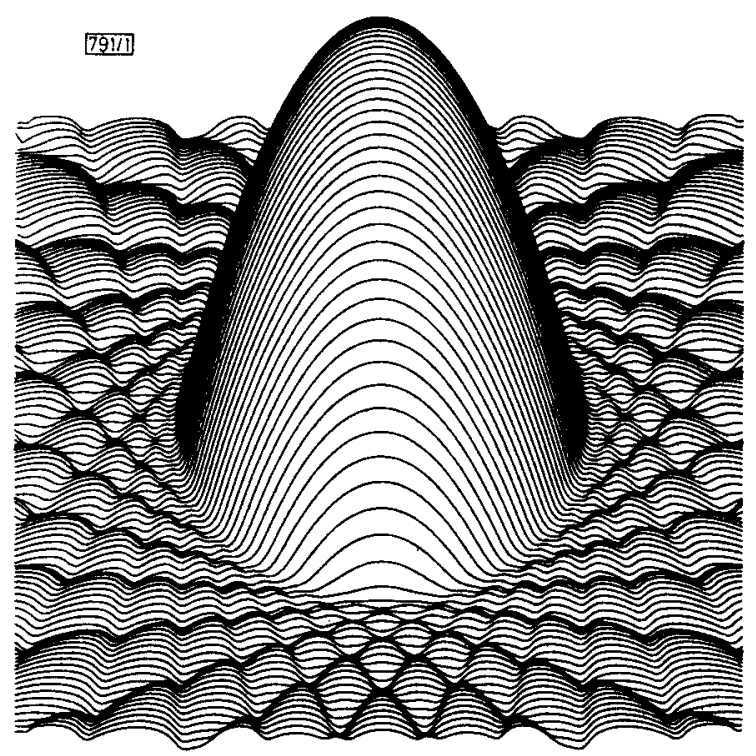

Fig. 1 Wave distribution arising for too small a number $N_{1}$ of integration points in eqn. 1

Preform core radius $=1 \mathrm{~mm}, \Delta=0.01$ explicit $\theta$-dependence in $\phi(\rho, \theta)$ and $g(z, \theta)$ disappears; in this ideal case, it can be shown ${ }^{4}$ that eqn. 1 reduces to Marcuse's equation: $:^{5}$

$$
\frac{n(r)-n_{0}}{n_{0}}=-\frac{1}{\pi} \int_{i}^{a} \phi(\rho) \frac{d \rho}{\sqrt{\left(\rho^{2}-r^{2}\right)}}
$$

where $\phi(\rho)=0$ for $\rho>a$.

From a numerical point of view, the integration of eqn. 3 is usually performed with a $\rho$-increment equal to the scan increment $\varepsilon$. If eqn. 1 is used instead of eqn. 3, and if likewise we take a $z$-increment equal to $\varepsilon$, then the number $N_{I}$ of increments in $\theta$
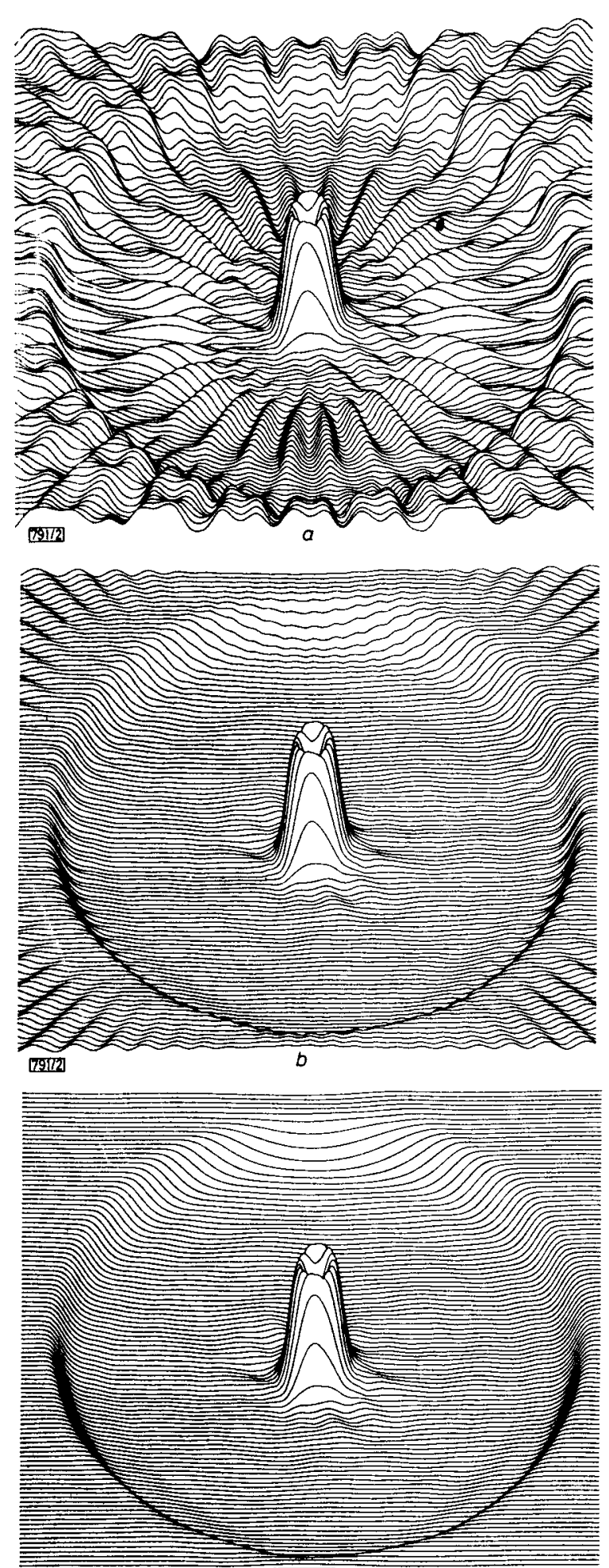

주일

Fig. 2 Effect of increasing, by interpolation, number $N_{I}$ of integration points in eqn 1

Preform core radius $=0.4 \mathrm{~mm}, \Delta=0.005$

$a$ No interpolation, $N_{1}=13$

$b$ Interpolation, $N_{l}=13 \times 3=39$

c $N_{t}=13 \times 9=117$; all wave effects disappear 
in the interval $(-\pi / 2, \pi / 2)$, can be evaluated from

$$
\varepsilon=\Delta z \simeq|r| \Delta \theta=|r| \frac{\pi}{N_{I}}
$$

For example, if $\varepsilon=10 \mu \mathrm{m}$ and $|r|=1 \mathrm{~mm}, N_{I} \simeq 314$. Practical values of $N$, range from 50 to 500 .

Actual preforms are never perfectly circular, and the use of eqn. 3 sometimes gives rise to an unacceptable error. ${ }^{4}$ In these cases, the explicit $\theta$-dependence of $g(z, \theta)$ in eqn. 1 must be retained, and each value of $\theta$ now corresponds to a different preform orientation. As the azimuthal profile variations are smooth, we have used interpolation: the $N$, necessary values of $g(z, \theta)$ are obtained from a number of projections $N_{\theta}$ which can be reduced to three in the case of nearly circular preforms. For a parabolic profile having a core ellipticity of $16 \%$, the reconstruction with only three projections was found ${ }^{4}$ to give a maximum index error, normalised to the total index difference, of $4.8 \%$. Even in the case of highly asymmetrical preforms, the azimuthal profile variations remain smooth, and the systematic use of interpolation considerably reduces the number of projections to be measured; for example, ${ }^{4}$ only about 20 projections are required in the case of a $75 \%$ ellipticity.

Results: A complete study of the influence on the reconstruction quality of the scan increment $\varepsilon$, the number $N_{I}$ of integration points in eqn. 1 and the number of projections actually measured is reported elsewhere. ${ }^{4} \mathrm{We}$ consider here the effect of choosing too small a value of $N_{I}$ and stress the improvement obtained by the systematic use of interpolation. Fig. 1 shows the simulated reconstruction of a perfectly circular preform with a parabolic index profile. $N_{1}$ was equal to only 13 , and we observe in Fig. 1 the typical wave distribution which arises when $N_{I}$ is too small. With $N_{I}=13 \times 2=26$, the waves are no longer observed. For variations in the profile shape more pronounced than in the parabolic case, the minimum value of $N_{t}$ giving an acceptable reconstruction of the cladding is increased. We now consider this effect in the case of a real preform which was measured with 13 projections (i.e. 13 scan directions in the interval $\left.\left(-90^{\circ}, 90^{\circ}\right)\right]$. In Fig. $2 a, N_{I}=13$, and we observe waves along 13 diameters corresponding to the 13 equally spaced preform orientations of the deflection measurement. The same effect is present in Fig. 1 but is perhaps more difficult to recognise. In Fig. $2 b$ and Fig. $2 c$, we have increased $N_{1}$ by interpolation: in Fig. $2 b$, with $N_{1}=13 \times 3=39$, the radial waves are only present in the substrate region, while, in Fig. $2 c$, with $N_{I}=13 \times 9=117$, they have entirely disappeared.
Conclusion: The importance of using a sufficient number of points for the integration involved in preform profile reconstruction has been demonstrated with both simulated and measured profiles. The systematic use of interpolation enables the optimum number of integration points to be obtained from the data corresponding to a few preform projections. This procedure reduces both the measurement time and the data storage requirements.

Acknowledgments: We wish to thank E. J. Tarbox, R. J. Mansfield and R. D. Birch for providing the preform used for Fig. 2, D. N. Payne for instigating the study and J. Baker for comments on the manuscript. P. L. Francois acknowledges financial support from the French Post Office.

P. L. FRANCOIS*

28th August 1981

I. SASAKI

M. J. ADAMS

Department of Electronics

University of Southampton

Southampton SO9 5NH, England

- On leave from CNET, Lannion, France

\section{References}

I CHU, P. L, and SABXEANG, C.: 'Nondestructive determination of refractive-index profile and cross-sectional geometry of opticalfibre preform', Electron. Lett., 1979, 15, (20), pp. 635-637

2 SASAKI, I. PAYNE, D. N., and ADAMS, M. J.: 'Measurement of refractive-index profiles in optical-fibre preforms by spatialfiltering technique', ibid., 1980, 16, (6), pp. 219-221

3 SASAKI, 1., FRANCOIS, P. L., and PAYNE, D. N.: 'Accuracy and resolution of preform index-profiling by the spatial-filtering method'. Proc. of 7 th European conference on optical communication, Copenhagen, sept., 1981, paper 6.4

4 FRANCOIS, P. L., SASAKI, I., and ADAMS, M. J.: 'Practical threedimensional profiling of optical fibre preforms' (to be published)

5 Marcuse, D.: 'Refractive-index determination by the focusing method', Appl. Opt., 1979, 18, pp. 9-13

6 OxosHI, T., and NISHIMURA, M.: 'Measurement of axially nonsymmetrical refractive-index distributions of optical fibre preforms by a triangular mask method', ibid., 1981, 20, pp. 2407-2411

$0013-5194 / 81 / 230876-03 \$ 1.50 / 0$

+ An analogous wave distribution has also been observed by other authors ${ }^{6}$ 